\title{
Encephalopathy and Cytotoxic Lesion of the Corpus Callosum Associated with Cytokine Storm in COVID-19: A
}

\section{Case Report}

\author{
Duyu A Nie, MD, PhD ${ }^{1,2 *}$ \\ ${ }^{1}$ Division of Pediatric Neurology and the Children's Neurodevelopment Center (CNDC), Hasbro Children's Hospital, USA \\ ${ }^{2}$ Department of Pediatrics, Neurology and Neurosurgery, Warren Alpert Medical School of Brown University, USA
}

\begin{abstract}
The neurological complications and neuroradiological spectrum of the SARS-CoV-2 infection (COVID-19 disease) in pediatric patients are increasingly recognized [1,2]; however, the pathophysiology of the central nervous system (CNS) involvement remains largely elusive. One hypothesis is that the activation of innate immunity and release of cytokines breaks down the blood brain barrier (BBB) and causes neuroinflammation. Here, I present a case of COVID-19 associated MIS-C (multisystem inflammatory syndrome in children) with neurological complications manifesting as encephalopathy and a cytotoxic lesion of the corpus callosum (CLOCC) on the brain MRI, in association with extremely elevated levels of circulating interleukin-6 (IL-6), and soluble interleukin-2 receptor (sIL2R). This case report has provided evidence in support of the cytokine hypothesis of the CNS complications in children diagnosed with COVID-19 associated MIS-C. Whereas our patient has well recovered following immunomodulatory therapy, the effects of acute neuroinflammation on long-term neurocognitive function require further evaluation.
\end{abstract}

\section{Keywords}

COVID-19, Cytokine storm, Brain MRI, Cytotoxic Lesion of the Corpus Callosum (CLOCC)

\section{Introduction}

Unlike adults, the vast majority of children with COVID-19 have mild symptoms in the acute, infectious phase of the disease, but a small minority of children becomes severely ill. MIS-C patients often present with multi-organ dysfunction that manifests late in the course of SARS-CoV-2 infection and most are positive for SARS-CoV-2 IgG antibodies [3]. This hyper inflammatory syndrome shares features with Kawasaki disease, toxic shock syndrome, and macrophage activation syndrome, primarily a cytokine-mediated process. On the contrary, some studies have questioned the role of cytokine storming in COVID-19-induced organ dysfunction [4,5]. The cytotoxic lesions of the corpus callosum (CLOCC) are associated with a heterogenous group of conditions. It typically manifests in children and can present with encephalopathy, nuchal rigidity, and seizures [6,7]. CLOCC is thought to be caused by a cytokine-mediated neuroinflammation as a result of the BBB disruption. While CLOCCs were previously reported in adult and pediatric patients with COVID-19 [8$10]$, so far there has not been strong laboratory evidence of a cytokine storming involved in the pathogenesis of COVID-19 associated CLOCCs.

\section{Case Report}

A previously healthy 12-year-old male presented to the emergency department (ED) after 3 days of fever, abdominal pain, nausea/vomiting and 2 days of frontal headache which he described as dull and achy. He was febrile at home to Tmax 102F. He was brought in after a witnessed episode of altered mental status where on waking, he was able to articulate words but did not make logical sense. He returned to baseline after approximately 5 minutes. In the ED, he was tachycardiac with intermittent fever. Subsequent labs at admission or

*Corresponding author: Duyu A Nie, MD, PhD, Division of Pediatric Neurology and the Children's Neurodevelopment Center (CNDC), Hasbro Children's Hospital, 335R Prairie Avenue, Suite 1A, Providence, Rhode Island, 02905, USA, Tel: 401-4444697

Accepted: July 03, 2021

Published online: July 05, 2021

Citation: Nie DA (2021) Encephalopathy and Cytotoxic Lesion of the Corpus Callosum Associated with Cytokine Storm in COVID-19: A Case Report. J Pediatr Neurol Neurosci 5(2):134-137 
hospital day 1 (HD1) showed WBC $10.1 \times 10^{9} /$ L (peak 20.7 on HD3), absolute lymphocyte count $0.3 \times 10^{9} / \mathrm{L}$ (nadir 0 on HD2), Na $127 \mathrm{mEq} / \mathrm{L}$, erythrocyte sedimentation rate 62 (peak 76 on HD4; ref range $0-15 \mathrm{~mm} / \mathrm{h})$, C-reactive protein 63.65 (peak 157.85 on HD4; ref range $0.00-10.00 \mathrm{MG} / \mathrm{L}$ ), Ferritin $386 \mathrm{ng} / \mathrm{mL}$ (peak 1030 on HD4; ref range 22-322 ng/mL), B-Type Natriuretic Peptide (BNP) 7 (peak 869.2 on HD3; ref range $0.0-33.3 \mathrm{pg} / \mathrm{mL}$ ), Fibrinogen 656 (peak 656; ref range 154-448 mg/dL), D-dimer 652 (peak 3082 on HD2; ref range 0-300 ng/mL), INR 1.8 (peak 3.9 on HD2), AST 32 IU/L (peak 132 on HD2), ALT 23 IU/L (peak 126 on HD2), Creatine kinase 39. Triglyceride peaked at $983 \mathrm{mg} / \mathrm{dL}$ on HD3 (ref range 30-89 $\mathrm{mg} / \mathrm{dL}$ ), and Troponin 1 peaked at 0.408 on HD3.

Adenovirus was detected from his nasopharyngeal swab; although the SARS-CoV-2 PCR (polymerase chain reaction) was not detected, he had positive total (IgG and IgM) antibodies against SARS-CoV-2. On day of presentation, EKG and echocardiogram were unremarkable. Initial chest and abdomen X-rays were normal. On HD2, the level of circulating Interleukin-6 (IL-6) was elevated at 1,460 pg/mL (Quest Diagnostics; ref range $<5.0 \mathrm{pg} / \mathrm{mL}$ ), and soluble IL2 receptor at $16,351 \mathrm{pg} / \mathrm{mL}$ (Viracor Eurofins; ref range 622-1,619 pg/ $\mathrm{mL}$ ). Repeat chest X-ray showed diffuse increasing interstitial thickening and multifocal patchy opacities in the lungs. A diagnosis of COVID-19 related MIS-C was made based on initial hyponatremia, lymphopenia, hepatitis, coagulopathy, elevated BNP, elevated inflammatory markers and cytokines. Subsequent hospital course was complicated by hypotensive shock needing fluid resuscitation and inotropes. Repeat echocardiogram was consistent with myocarditis. He was intubated on HD3 for hypoxic respiratory failure, with CT and $\mathrm{CT}$ angiogram showing diffuse ground glass opacities in the lungs, bibasilar consolidation and bilateral pleural effusions (shown in Figure 1). Moderate ascites and segmental colitis were also evident. He had negative non-contrast CT head twice. Blood and urine cultures remained no growth.
Neurology was consulted for abnormal head movements described as intermittent neck extension. Differential diagnosis included diaphragmatic irritation (i.e., hiccups) versus myoclonus, rather than an epileptic nature. Neurologic exam was notable for diffuse hyperreflexia yet negative Babinski signs. Due to a concern for ADEM (Acute disseminated encephalomyelitis), MRIs of the brain and cervical spine with and without contrast were obtained. While the spine MRI was unremarkable, the brain MRI showed a restricted diffusion area in the splenium of the corpus callosum without contrast enhancement, consistent with a Cytotoxic Lesion of the Corpus Callosum (CLOCC) [11] (shown in Figure 2). Lumbar puncture was not attempted due to coagulopathy. Patient was treated with two rounds of intravenous immunoglobulin (IVIG, $2 \mathrm{mg} / \mathrm{kg}$ ), IV methylprednisolone $(1 \mathrm{mg} / \mathrm{kg}$ every 6 hours), heparin drip initially then transitioned to Lovenox. He was discharged on low dose aspirin, oral prednisone taper, and to continue PT/OT. He never required anti-seizure drugs during the 10 days hospitalization course. On 6-week followup, he had reassuring cardiac assessment, normal EKG and echocardiogram. The ongoing headaches were consistent with migraine. His involuntary jerking movements were suggestive of tics. Otherwise, he had recovered well.

\section{Discussion}

Our patient has probably a concurrent adenovirus infection; however, his clinical evolution and neurological complications are not typical for adenovirus infection [12]. CLOCC is a non-specific finding associated with a variety of etiologies [13], and has been reported in adult and pediatric patients with COVID-19, but in children predominantly observed in MIS-C patients $[2,8,10]$. A hypothesized mechanism of this non-ischemic lesion involves the vulnerability of the oligodendrocytes-rich splenium to markedly increased levels of cytokines and extracellular glutamate [11]. High levels of circulating IL-6 and sIL2R seen
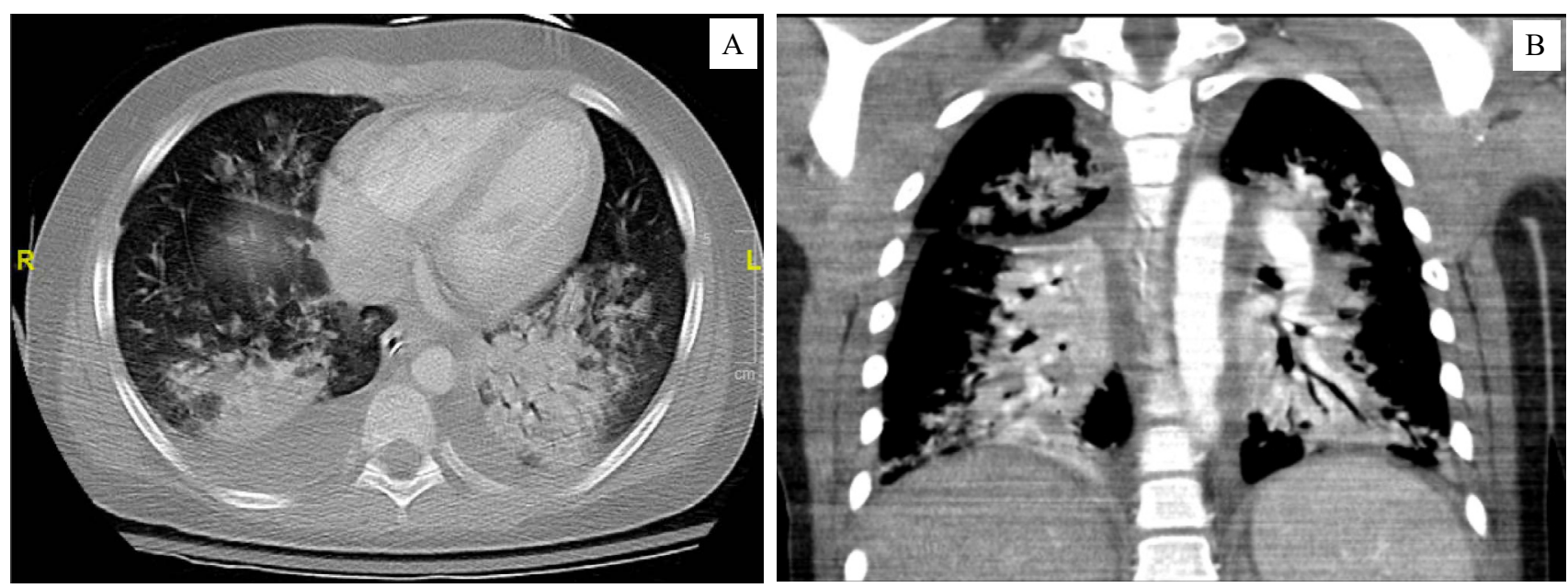

Figure 1: Chest/Abdomen CT and CT angiogram with intravenous contrast (A- axial; B- coronal) shows diffuse multifocal ground glass opacities throughout the lungs with bibasilar consolidation and bilateral pleural effusions. Findings are compatible with Covid-19 associated pneumonia or ARDS (Acute Respiratory Distress Syndrome). 

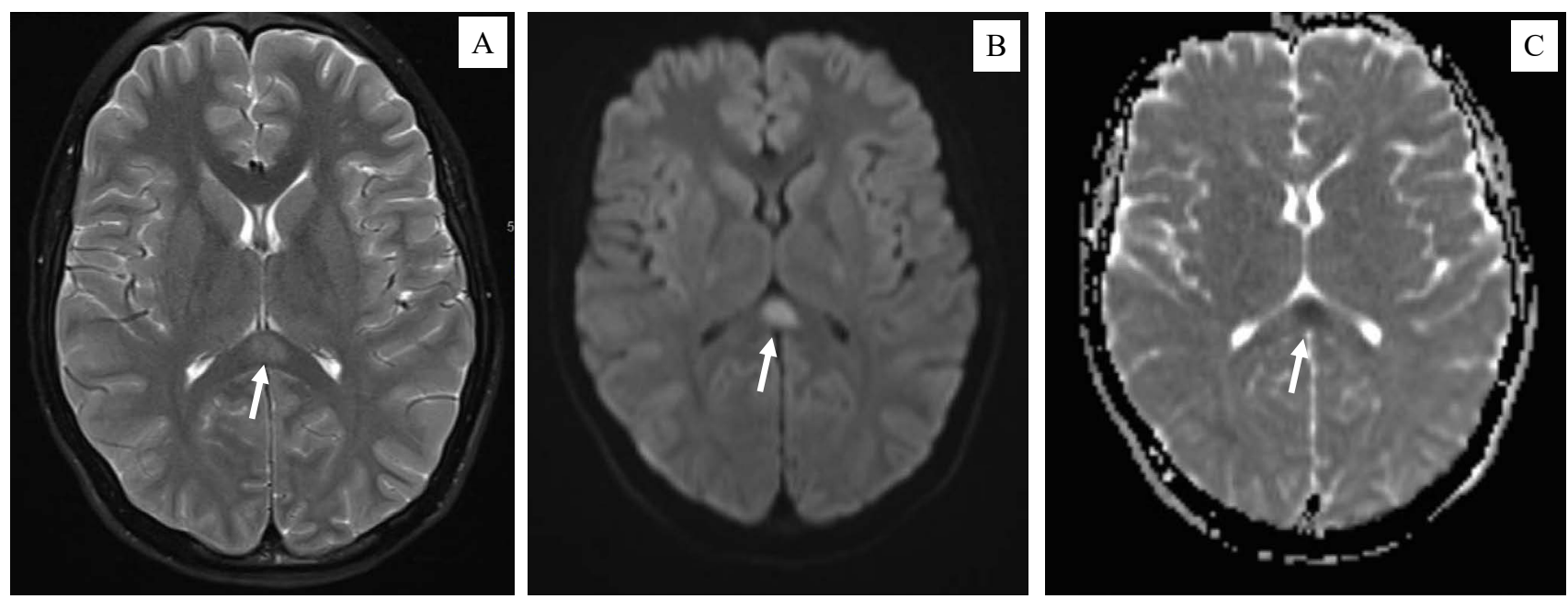

Figure 2: Axial MR images demonstrate a focal hyperintensity lesion, as denoted by the arrows, A) In the splenium of the corpus callosum on T2WI; B) With associated restricted diffusion on DWI and C) ADC map.

in our patient have provided evidence of cytokine storming and T-cell activation in the pathogenesis of COVID-19 related encephalopathy and CLOCCs. Indeed, several studies have shown a positive association of IL-6 and SIL2R levels with the disease severity, at least in adult patients with COVID-19 $[14,15]$. Notably, our patient recovered well following the immunomodulatory therapy, not requiring cytokine antagonists such as Tocilizumab. This observation aligns with the overall outcome discrepancy between adult and pediatric COVID-19 patients.

Whether there is a causative cytokine level in the cerebrospinal fluid (CSF) in COVID-19 patients presenting with CNS complications remains to be investigated. Interestingly, literature search has revealed no case report of FIRES (Febrile Infection-Related Epilepsy Syndrome) after COVID-19 infection despite the cytokine storming and innate immunity activation. Our patient has recovered during acute phase following immunomodulatory therapy; however, the effects of cytokine storming and neuroinflammation on long-term neurodevelopmental outcomes and even risk for chronic epilepsy require a longitudinal follow-up.

\section{Declaration of Conflicting Interest}

The author has no potential conflicts of interest to disclose.

\section{Funding}

The authors received no financial support for the research, authorship, and/or publication of this article.

\section{References}

1. LaRovere KL, Riggs BJ, Poussaint TY, et al. (2021) Neurologic Involvement in Children and Adolescents Hospitalized in the United States for COVID-19 or Multisystem Inflammatory Syndrome. JAMA Neurol 78: 536-547.

2. Lindan CE, Mankad K, Ram D, et al. (2021) Neuroimaging manifestations in children with SARS-CoV-2 infection: a multinational, multicentre collaborative study. Lancet Child Adolesc Health 5: 167-177.
3. Henderson LA, Canna SW, Friedman KG, et al. (2020) American College of Rheumatology Clinical Guidance for Multisystem Inflammatory Syndrome in Children Associated With SARSCoV-2 and Hyperinflammation in Pediatric COVID-19: Version 1. Practice Guideline 72: 1791-1805.

4. Leisman DE, Ronner L, Pinotti R, et al. (2020) Cytokine elevation in severe and critical COVID-19: A rapid systematic review, metaanalysis, and comparison with other inflammatory syndromes. Lancet Respir Med 8: 1233-1244.

5. Chen LYC, Hoiland RL, Stukas S, et al. (2020) Confronting the controversy: Interleukin-6 and the COVID-19 cytokine storm syndrome. Eur Respir J 56: 2003006.

6. Parri N, Lenge M, Buonsenso D (2020) Children with Covid-19 in Pediatric Emergency Departments in Italy. N Engl J Med 383: 187-190.

7. Hashimoto Y, Takanashi J-i, Kaiho K, et al. (2009) A splenial lesion with transiently reduced diffusion in clinically mild encephalitis is not always reversible: A case report. Brain Dev 31: 710-712.

8. Gaur P, Dixon L, Jones B, et al. (2020) COVID-19-Associated Cytotoxic Lesions of the Corpus Callosum. AJNR Am J Neuroradiol 41: 1905-1907.

9. Moreau A, Ego A, Vandergheynst F, et al. (2021) Cytotoxic lesions of the corpus callosum (CLOCCS) associated with SARSCoV-2 infection. J Neurol 268: 1592-1594.

10. Edjlali M, Le Gal A, Louvet M, et al. (2020) Teaching Neurolmages: Cytotoxic lesions of the corpus callosum in encephalopathic patients with COVID-19. Neurology 95: 1021-1022.

11. Starkey J, Kobayashi N, Numaguchi Y, et al. (2017) Cytotoxic Lesions of the Corpus Callosum That Show Restricted Diffusion: Mechanisms, Causes, and Manifestations. Radiographics 37: 562-576.

12. Schwartz KL, Richardson SE, MacGregor D, et al. (2019) Adenovirus-Associated Central Nervous System Disease in Children. J Pediatr 205: 130-137.

13. Rasmussen C (2020) COVID-19 and Involvement of the Corpus Callosum: Potential Effect of the Cytokine Storm? AJNR Am J Neuroradiol 41: 1625-1628. 
14. Zeng $\mathrm{Z}, \mathrm{Yu} \mathrm{H}$, Chen $\mathrm{H}$, et al. (2020) Longitudinal changes of inflammatory parameters and their correlation with disease severity and outcomes in patients with COVID-19 from Wuhan, China. Crit Care 24: 525.
15. Azmy V, Kaman K, Tang D, et al. (2021) Cytokine Profiles Before and After Immune Modulation in Hospitalized Patients with COVID-19. J Clin Immunol 41: 738-747.

DOI: $10.36959 / 595 / 422$ 